\title{
NONSTATIONARY PROCESSES AND MAGNETIC FIELD GENERATION IN BINARY STELLAR SYSTEMS
}

\author{
A. Z. DOLGINOV \\ Physical Technical Institute, Academy of Sciences, Leningrad, U.S.S.R.
}

\begin{abstract}
This note gives introductory remarks to the three papers mentioned in the references.
The radiation flux variations of the stars in some cases can be connected with the change of the heat transfer in their convective zones. It can be due to the corresponding magnetic field change. This process leads to an equilibrium state if the field is generated by convective motion. If the generation process is determined by some outer forces, the convective motion can be strongly disturbed by the field. In binary stellar systems there are tidal forces which may be responsible for the field generation. If the star is a member of a binary system and has its axis of rotation inclined to the orbital plane then there are tidal flows of complicated spiral symmetry inside the star. Such symmetry is favorable for magnetic field generation. Although the tidal deformations are almost elastic and the shape of the body comes back to its previous configuration, the elements of its substance do not come back to their previous relative positions. The magnetic lines frozen in the matter are elongated and entangled more and more which leads to the magnetic flux increase. The dissipating part of the tidal energy is sufficient for the magnetic field generation. The estimation of characteristic time of the field increase gives $\tau \approx 3 L^{2} \omega_{t} v_{t}^{-2}$, where $L, \omega_{t}$ and $v_{t}$ are the average values characterizing the size of the generation region, and the frequency and velocity of the tidal flows. In sufficiently close binary systems (both outside and inside the Roche region) the increasing time can be small, $\sim 10-10^{3} \mathrm{yr}$. In this case strong disturbances of convective heat transfer and thermal balance of the star are possible. The tidal flows are spread throughout the whole stellar volume and the field is also generated in the volume although, in the convective zone, the field can turn out to be very irregular. Such a field would be difficult to observe. Only if convection is suppressed does the regular field appear in the outer layers. The radical disturbance of the thermal balance can lead to essential changes in the radiation flux and in some cases to explosive phenomena like flares or outbursts. The explosion changes both the field and the flow distributions drastically and the generation process starts from the beginning. It cannot be excluded that the explosions of the nova-like stars are connected with this valve mechanism.
\end{abstract}

\section{References}

Dolginov, A. Z.: 1973, Pisma v JETP 18, 67.

Dolginov, A. Z.: 1974, Astron. Zh. U.S.S.R. 51, 388.

Dolginov, A. Z. and Yakovlev, D. G.: 1975, Astrophys. Space Sci., in press. 


\section{DISCUSSION}

V. M. Tomozov: Did Dr Dolginov consider the processes of magnetic field damping?

A. $Z$. Dolginov: Undoubtedly, the damping of the magnetic field was taken into consideration as the term with conductivity was included into the equation of the magnetic field.

S. D. Sinvhal: It was stated that assumption of the inclination of the rotation axes of the components of a binary system to the plane of rotation is necessary to give rise to non-stationary processes in the stars. Are there any limits to this inclination?

$A$. $Z$. Dolginov: The limits are determined by the parameters of the system. They arise because of a $\sin \beta$ term in the treatment of the problem. For the system discussed, $\beta=45^{\circ}$. 